\title{
La lucha contemporánea por el espacio en la obra de Carl Schmitt
}

Pablo Beytía

Departamento de Filosofía, Universidad de Chile

\section{Introducción}

Tanto en Tierra y Mar (1942) como en El Nomos de la Tierra (1950), Carl Schmitt exploró una tarea compleja para la historiografía y comúnmente difícil de defender: la elaboración de estadios o etapas claramente identificables en la historia universal. Desde el comienzo, esta tarea estuvo respaldada por fundamentos antropológicos, que el jurista alemán planteó en Tierra y Mar:

«El hombre es un ser terrestre, un ente terrícola. Se sostiene, camina y mueve sobre la tierra firme. Ella es el punto de partida y de apoyo. Ella determina sus perspectivas, sus impresiones y su manera de ver el mundo. No sólo su horizonte sino también su modo de andar, sus movimientos y su figura son los de un ser que nace y vive sobre la tierra» (Schmitt 1952, p. 7).

En completa sintonía con esta visión «terrícola» de lo humano, Schmitt pensaba que el fundamento de cada época histórica podía descubrirse en el nomos de la tierra, un tipo específico de división espacial que se encuentra en la base de diversos fenómenos socialmente primarios, como la coexistencia de los pueblos, el derecho, la política y la guerra. El nomos sería una especie de estructura estructurante, una gramática espacial que condiciona los eventos históricos.

A partir de este concepto, Schmitt desarrolló una teoría de la evolución histórica. Esta afirmación podría parecer excedida, en especial si se considera que Tierra y Mar fue en su origen un libro infantil que Schmitt contaba a su hija Anima antes de dormir (Voigt 2012: 13). Lo cierto, sin embargo, es que en ambas obras — producidas con un desfase menor a una década-, el jurista alemán desarrolló genuinos aportes a la filosofía de la historia y un modelo más o menos sistemático del cambio social a gran escala. Ambos escritos distinguen — con la rigurosidad de quien acostumbra a trabajar con leyes- conceptos, mecanismos y estadios de cambio histórico.

Lo problemático de la propuesta de Schmitt, sin embargo, no está en lo que estas obras dicen, sino en lo que callan. Si bien ellas contienen una interpretación sólida del pasado y una descripción de los procesos claves en el presente, no incorporan una apuesta clara sobre los nuevos senderos que persigue actualmente la evolución histórica. «Un nuevo nomos de nuestro planeta surge incontenible e inevitable — sostiene Schmitt (1952, p. 113)—. Reclámanlo las nuevas relaciones entre el hombre y los nuevos elementos; la alteración de medidas y proporciones lo impone». Pero, con la misma seguridad que el jurista denota un cambio de época en los albores del siglo XX, sostuvo en 1942 que era demasiado pronto como para diagnosticar seriamente la dirección de estas transformaciones históricas, o, en

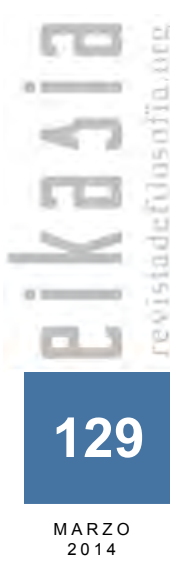


otras palabras, el nuevo elemento ${ }^{1}$ - fuego o aire — en el que se sostendrían las luchas por el ordenamiento espacial planetario.

Queda claro en su obra, que estaría comenzando un nuevo estadio histórico; lo incierto, no obstante, sería la estructura espacial que vendría a definir esta etapa. Al menos en sus dos grandes escritos destinados al concepto de nomos, sólo aparecen insinuaciones o sondeos. En este ensayo se postulará que para Schmitt el estadio actual de la historia universal remite a la coexistencia de dos procesos interrelacionados: en la dimensión nómica, existiría una lucha por la apropiación industrial de los grandes espacios, mientras que en la dimensión imaginaria, se establecería una conciencia colectiva que entiende el espacio como un campo de fuerzas donde el hombre despliega su energía, su actividad y su esfuerzo.

Para defender fundadamente esta tesis, será necesario examinar una serie de nociones y mecanismos teóricos introducidos en la obra de Schmitt. En la primera sección, se explorará el concepto de nomos como elemento constituyente del ordenamiento espacial y social, vinculado estrechamente al derecho, la política y la guerra. Posteriormente, se argumentará que para Schmitt el surgimiento de estadios históricos se basa en la gestación de dos elementos interrelacionados: un nomos y una imagen colectiva del espacio. Luego se mostrará cómo, para el jurista alemán, se desarrollaron estos procesos de cambio en la gran revolución espacial de los siglos XVI y XVII. Finalmente, se indagará en los cambios producidos a partir del siglo XX, que hacen suponer a Schmitt la gestación de un nuevo nomos y una nueva imagen del espacio. En esta última sección se mostrará, a partir de algunos escritos de posguerra, que el orden mundial contemporáneo fue entendido por él fundamentalmente como una lucha por los espacios industriales - lo que cuestiona otras versiones posibles, como la pugna por los espacios aéreos o por el control del fuego-.

\section{En el origen fue el nomos}

Entender el concepto nomos y su vinculación esencial con aspectos primarios de la vida social — tales como el derecho, la política y la guerra-, es fundamental para entender la perspectiva schmittiana del cambio histórico. Dicho concepto no es utilizado por Schmitt en el sentido de «regla» — noción predominante ya incluso en los escritos de Platón-, sino que en una versión más primitiva, originada también en la Antigua Grecia. En efecto, tanto Heráclito como Píndaro indicaron que «todas las regulaciones ulteriores, escritas o no escritas, toman su fuerza de la medida interna de un acto primitivo constitutivo de ordenación del espacio» (Schmitt 2002, p. 45). Este acto creador sería el nomos, definido por Schmitt (2002, p. 31) como «la primera medición en la que se basan todas las mediciones ulteriores», es decir, «la primera toma de la tierra como primera partición y división del espacio».

\footnotetext{
${ }^{1}$ Cuando en este artículo se mencione la palabra «elemento» o «elementos», normalmente implicará una referencia a la teoría griega y materialista de los cuatro elementos - tierra, agua, aire y fuego-, de la cual participaron filósofos como Heráclito, Aristóteles, Anaxímenes, Diógenes y Empédocles (Aristóteles 2003).
} 
Todo evento posterior - ya sea consecuencia, ampliación o nueva distribución — sería continuación o modificación de este acto primigenio de ordenamiento espacial.

Dicho acto constituyente no es considerado por Schmitt como contingente - a pesar de que su forma sí pueda variar históricamente- La presencia de un nomos es de hecho inevitable, debido a que toda coexistencia de pueblos se basa en una división especifica del espacio. En palabras del jurista:

«Mientras la historia del mundo no esté concluida, sino que se encuentre abierta y en movimiento, [...] también surgirá, en las formas de aparición siempre nuevas de acontecimientos históricos universales, un nuevo nomos. Así pues, se trata para nosotros del acto fundamental divisor del espacio, esencial para cada época histórica [...]. Este es el sentido en el que se habla aquí del nomos de la tierra; pues cada nuevo período y cada nueva época de la coexistencia de pueblos, imperios y países, de potentados y potencias de todo tipo, se basa sobre nuevas divisiones del espacio, nuevas delimitaciones y nuevas ordenaciones espaciales de la tierra» (Schmitt 2002, pp. 45-46).

Con estas palabras, Schmitt sitúa al nomos como una categoría previa a otras categorías, una división material sobre la cual se pueden asentar otras divisiones materiales y diversos imaginarios colectivos sobre el espacio. De manera metafórica, aunque bastante precisa, sugiere Schmitt (1952, p. 90) que el nomos actuaría como «ley orgánica». Para un jurista esto significa esencialmente dos cosas: por un lado, que sería un requisito para la regulación de otros fenómenos, y por el otro, que tendría requerimientos extraordinarios para su gestación y derogación.

A grandes rasgos, el establecimiento de un nomos de la tierra podría enlazarse, en la obra schmittiana, al menos con el surgimiento de tres fenómenos sociales primarios que vale la pena introducir: derecho, unidad política y guerra.

En el primer corolario de El Nomos de la Tierra, Schmitt (2002, pp. 3-4) sostiene un vínculo triple entre tierra aquello que el nomos divide - y derecho. Para iniciar — indica el jurista-, la tierra contiene al derecho en sí misma, como premio del trabajo: cuando las personas poseen tierra fértil, la cantidad de esfuerzo en la siembra y el cultivo es recompensada por el crecimiento de la cosecha; de tal modo, hay cierta justicia y derecho asociados al grado de trabajo. En segundo lugar, la tierra revela al derecho en sí misma, como límite firme: cuando el suelo es trabajado por el hombre, se hacen visibles ciertas divisiones a partir de las fronteras entre campos, praderas o bosques, las cuales evidencian medidas y reglas de cultivo sobre las cuales se desarrolla el trabajo humano. Finalmente, la tierra lleva al derecho sobre sí misma, como signo público del orden: sobre su superficie existen cercados, muros y casas, revelando la ordenación y el asentamiento de la convivencia humana; sobre ella se hacen visibles la familia, la casta, la propiedad, la posición social e incluso las formas de poder y dominio. 
A su vez, el acto primigenio de división del espacio podría vincularse con la demarcación schmittiana de la unidad política. Después de todo, «el sentido de la distinción amigo y enemigo - aquella que permite, según Schmitt, distinguir las acciones y motivos políticos de otras acciones y motivos - es marcar el grado máximo de intensidad de una unión o separación, de una asociación o disociación» (Schmitt 2006, p. 57). Esto significa, en otras palabras, que la distinción política se superpone a la distinción espacial, aportando intensidad a la unión/separación de terrtorios ya demarcados por el nomos. Esto sucedería muy claramente en el Estado, ya que éste es considerado por Schmitt como una unidad política territorialmente demarcada ${ }^{2}$, que además decide como un todo acerca del amigo y enemigo (Schmitt 2006, p. 49 y 59). El Estado, entonces, sería el ejemplo más evidente de cómo la demarcación territorial contemporánea se solapa con la distinción amigo/enemigo, o, en otras palabras, de cómo en nuestra época lo nómico se ha transformado en un problema político.

Finalmente - y muy relacionado con el punto anterior-, la posesión de tierra implicada en el concepto schmittiano de nomos tiene una vinculación lógica con la guerra. Como se ha recalcado previamente, el concepto de Estado ensayado por Schmitt asocia la delimitación espacial con la decisión sobre amistad y enemistad. Sin embargo, en esta perspectiva el enemigo no representa cualquier adversario o competidor, sino «sólo un conjunto de hombres que siquiera eventualmente, esto es, de acuerdo con una posibilidad real, se opone combativamente a otro conjunto análogo» (Schmitt 2006, p.58) En otras palabras, lo propio del enemigo — que en el caso del Estado refiere a todos quienes quedan fuera del límite espacial demarcado nómicamente - sería su eventual posición de guerra, entendida como «lucha armada entre unidades políticas organizadas» (Schmitt 2006, p. 62)

\section{Dos procesos revolucionarios: nomos y conciencia colectiva}

Entendiendo, desde la terminología schmittiana, el vínculo entre nomos y algunos conceptos sociales primarios como derecho, unidad política y guerra-, es posible indagar más expeditamente en el modo en que Schmitt concibe las transformaciones en la historia universal.

En primer lugar, el jurista señala que el cambio histórico está fuertemente asociado a la transformación sucesiva de los espacios; de hecho, para él «todo ordenamiento fundamental es un ordenamiento espacial» (1954, p. 74), situación que lo lleva a interpretar la historia universal específicamente como una historia de conquistas (1954, p. 76). Su argumento completo, queda explícito en el tercer párrafo del capítulo X de Tierra y Mar:

«Las fuerzas y energías históricas no aguardan, sin embargo, a la ciencia, como no esperó Cristóbal Colón a Copérnico. Cada vez que mediante un nuevo impulso de ellas son incorporados nuevas

\footnotetext{
${ }^{2}$ A pesar de que lo considera sólo una aproximación, Schmitt (2006, p. 49) define el Estado como «el status político de un pueblo organizado en el interior de unas fronteras territoriales».
} 
tierras y mares al ámbito visual de la conciencia colectiva de los hombres, transfórmanse también los espacios de su existencia histórica. Surgen entonces nuevas proporciones y medidas de la actividad histórico-política, nuevas ciencias, nuevas ordenaciones, vida nueva de pueblos nuevos o que vuelven a nacer. El ensanchamiento puede ser tan grande, tan sorprendente, que cambien no sólo proporciones y medidas, no únicamente el horizonte externo del hombre, sino también la estructura del concepto mismo de espacio. Se puede hablar entonces de revolución espacial. Las grandes transformaciones históricas suelen ir acompañadas, en verdad, de una mutación de la imagen del espacio» (Schmitt 1952, p 58).

Este párrafo es fundamental, ya que en él Schmitt identifica y relaciona los principales procesos que caracterizan a las revoluciones espaciales. Según su visión, las fuerzas y energías históricas —nociones que lamentablemente no profundiza - impulsarían la inclusión de nuevos espacios visuales, los cuales se integrarían en la conciencia colectiva de los hombres, transformando las proporciones y medidas de la actividad histórica; si este proceso es tan radical que llega a provocar un cambio en la imagen del espacio, se trataría entonces de una revolución espacial.

Dos son entonces los principales procesos que se identifican en esta revolución: primero, la apropiación nomistica del espacio — este paso inicial en donde se produce la «inclusión de nuevas tierras y mares al ámbito visual»—, y luego, la transformación de la conciencia colectiva - en donde se establecen, a nivel social, tanto las «nuevas proporciones y medidas de la actividad histórico-política» como la naciente «estructura del concepto mismo de espacio»-

Es notable que, para describir este proceso, Schmitt otorgue un lugar principal a la «conciencia colectiva». Este concepto evoca la sociología de Émile Durkheim y refiere al conjunto de representaciones grupales en donde se expresa cómo la sociedad se piensa a sí misma en relación con los objetos que la afectan (Durkheim 2008, pp. 2223). Esta mentalidad común, funcionaría con leyes diferentes a las de los estados psicológicos individuales y, en tanto hecho social, podría ejercer una influencia coercitiva en las conciencias particulares (Durkheim 2008, p. 27). Ello sugiere, que Schmitt ve en el proceso de revolución espacial un fenómeno social estructural, en el sentido de que podría distinguirse claramente de las voluntades humanas e incluso funcionar coercitivamente sobre ellas.

El proceso, aún no revolucionario, de transformación en las proporciones y medidas históricas debido a la inclusión de nuevos espacios visuales, es ejemplificado por Schmitt (1952, pp. 59-65) a partir de tres sucesos históricos: las conquistas de Alejandro Magno, la expansión del Imperio Romano de Julio César y las Cruzadas medievales. Producto de las primeras, en Grecia se habrían producido numerosas invenciones técnicas - como las máquinas de guerra de Arquímedes—, físicas — los cálculos del Ecuador y de la curvatura de la Tierra realizados por Eratósthenes - y matemáticas — como la geometría euclidiana-. Por su parte, la expansión del Imperio Romano habría condicionado que Séneca, por ejemplo, tuviera una nueva conciencia espacial planetaria. A

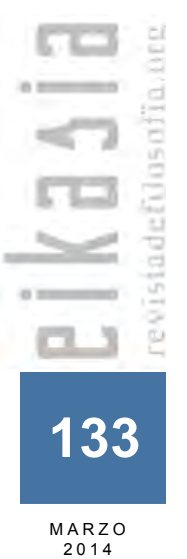


consecuencia de las Cruzadas, finalmente, surgieron universidades, renació el Derecho Romano, emergió la arquitectura Gótica y se anunció la formación del Estado moderno.

Si bien estos procesos produjeron enormes transformaciones espaciales, Schmitt no considera que hayan sido revolucionarios, principalmente porque no cambiaron la imagen colectiva del espacio, limitándose normalmente a un acotado círculo de sabios. Algo muy distinto sucedió entre los siglos XVI y XVII, en donde se habría producido «la más honda y trascendental transformación de la imagen planetaria del mundo de que tenemos noticia en la Historia Universal» (Schmitt 1952, p. 66).

\section{La gran transformación imaginaria}

Lo característico de la revolución del siglo XVI y XVII — señala Schmitt—, no tiene que ver con la dilatación del horizonte geográfico que se produjo al momento en que los navegantes europeos descubrieron tierras y mares. ${ }^{3} \mathrm{Sin}$ restarle importancia a las hazañas de Vasco da Gama o Cristóbal Colón, el jurista plantea un cambio más decisivo provocado en la conciencia colectiva: «el agrandamiento del Cosmos en sí y la idea de un infinito espacio vacío» (Schmitt 1952, p. 67).

Si bien, por vez primera en la historia, podía tener el hombre en su mano la representación completa de la esfera terrestre, ese proceso no habría transformado — sino ampliado el horizonte de - la imagen social del espacio. En contraste, la construcción conceptual gestada por intelectuales como Copérnico, Bruno, Galileo, Kepler y Newton - que luego fue apoyada y difundida por la Ilustración—, sí era revolucionaria. Esta nueva imagen del espacio, suponía que «equilibradas las fuerzas de atracción y repulsión, muévense los astros según leyes de gravedad en un espacio vacío» (Schmitt 1952, p. 68).

En este punto, creo que la perspectiva de Schmitt podría equipararse con la que posteriormente defendió Hannah Arendt. Para la politóloga alemana, la Época Moderna habría estado determinada por tres transformaciones de índole espacial: el descubrimiento de América — con la consiguiente exploración de toda la Tierra-, la expropiación de terrenos eclesiásticos y monásticos en la Reforma, y «el desarrollo de una nueva ciencia que considera la naturaleza de la Tierra desde el punto de vista del universo» (Arendt 2005, p. 277). El punto decisivo, es que Arendt también considera revolucionario el cambio en la imagen espacial del mundo, y coincide con Schmitt al dar un papel vital, en el desarrollo de esta transformación, a la emergente ciencia moderna. Para la politóloga alemana, la Época Moderna «sólo fue posible porque al principio la antigua dicotomía entre Tierra y cielo se abolió y se efectuó una unificación del universo» (Arendt 2005, p. 289); este cambio en la cosmovisión espacial, habría

\footnotetext{
${ }^{3}$ Esta situación, como ha mostrado recientemente Ferguson (2012, p. 69-77), había sido enormemente avanzada en China a partir de los viajes desarrollados por Zhen He entre 1405 y 1424.
} 
sido propiciado por el uso científico del telescopio (Arendt 2005, pp. 285-294).

Schmitt consideraba que, en éste período, la transformación imaginaria del espacio fue "de signo total», y a ello contribuyeron todas las corrientes intelectuales emergentes en Europa entre los siglos XVI y XVII —el Renacimiento, el Humanismo, la Reforma, la Contrarreforma y el Barroco- (Schmitt 1952, p. 72-73).

«No es excesivo —indicó el jurista — afirmar que toda clase de fuerzas creadoras humanas, arte, ciencia y técnica, han participado de aquel nuevo sentido espacial. Los grandes cambios de la tierra en lo geográfico no son sino el aspecto externo de la honda transformación que expresa la trascendental palabra «revolución espacial». Todo lo que se ha caracterizado como supremacía racional del europeo y del racionalismo occidental, surge entonces con impulso irresistible» (Schmitt 1955, p. 70).

Este pasaje da luces acerca de cómo concibe Schmitt la dirección o causalidad en los procesos históricos. En una primera etapa, la revolución espacial habría estado escoltada por diversas creaciones - artísticas, científicas, técnicas o de otra índole - producidas en los siglos XVI y XVII. Una vez engendrada esta trasformación imaginaria del sentido espacial - y su correspondiente manifestación geográfica-, ella serviría como base para el racionalismo occidental - y no a la inversa- Es altamente probable que, con esta última declaración, Schmitt haya intentado recalcar que los procesos nomísticos e imaginarios por él descritos eran más esenciales y primarios que los considerados por Max Weber (2004), quien célebremente interpretó el proceso de modernización occidental utilizando la racionalización social como clave hermenéutica.

Por otra parte, en ese mismo pasaje Schmitt reconoce que los cambios geográficos son una manifestación externa de la transformación espacial revolucionaria. Ello no debe interpretarse como que fueran accesorios, ya que toda conciencia del espacio - y también la emergente en el siglo XVI - se sustenta en un determinado nomos. Ello queda muy claro en Tierra y Mar, cuando el jurista señala: «todo cambio o variación notable de la imagen de la tierra va unido a cambios políticos universales, a una nueva distribución del globo, a una nueva conquista de territorios» (Schmitt 1952, p. 74). Eso explica que la gran revolución imaginaria producida en estos siglos, haya acarreado una conquista igualmente asombrosa - la que fue iniciada por Castilla y Portugal, y seguida prontamente por Holanda, Francia, Inglaterra y Dinamarca-.

Los procesos políticos y guerreros de esta época, estuvieron vinculados a la nueva imagen espacial del mundo, pero también a la transformación del derecho. Como se ha señalado, Schmitt reconoce en la tierra un fundamento decisivo del orden jurídico; por ese motivo, el Ius Publicum Europaeum difícilmente podría haber quedado inmune a las numerosas conquistas territoriales. Efectivamente, desde finales del siglo XV, el Derecho de Gentes tuvo importantes cambios ligados al reparto de los nuevos espacios mundiales — es decir, al proceso de establecer un

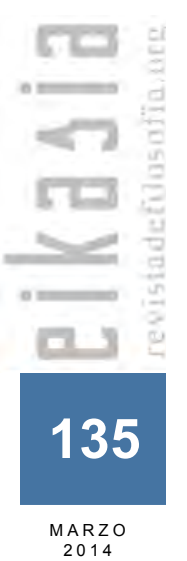


nomos para los continentes en disputa- Si bien Europa se encontraba en una época de lucha sangrienta, sorpresivamente sus pueblos mantuvieron un consenso: «considerar el territorio no europeo como suelo colonial, es decir, como objeto de conquista y explotación» (Schmitt 1952, p. 78). Este acuerdo jurídico sería fundamental para entender el cambio de estadio histórico, dado que habría dado inicio a la lucha por el nuevo ordenamiento espacial - el nuevo nomos de la tierra - que Schmitt fecha simbólicamente en 1493, cuando el Papa Alejandro VI establece, a partir de la Bula Inter Caetera, una división territorial del «nuevo mundo» a favor del reino de Castilla y Aragón (Schmitt 1952, p. 80).

En el crepúsculo del siglo XV, entonces, habría comenzado la lucha por los nuevos espacios. Lo decisivo es que ella, desde el comienzo, incorporó un elemento nuevo de apropiación y combate: el mar. Schmitt es muy claro al distinguir dos tipos de órdenes espaciales, que en su diferencia contendrían la esencia del primer ordenamiento espacial planetario: la tierra firme, por un lado, consistía en la división de territorios estatales; el mar, en contraste, era «libre» en el sentido de que no conocía la soberanía estatal. Ese era el hecho fundamental sobre el que se desarrolló, según el jurista alemán, el derecho internacional europeo de los últimos siglos (Schmitt 1952, p. 90).

El gran triunfador de esta lucha por el ordenamiento espacial mundial, habría sido Inglaterra. A pesar de su incorporación relativamente tardía en el combate por nuevas tierras — si se le compara con Castilla o Portugal—, esa nación lideró la partición de los espacios mundiales desde el siglo XVI, justamente por haber entendido el cariz de la nueva dimensión de lucha. Si bien en la historia occidental existieron pueblos fluviales y marítimos -e incluso, siguiendo a Jacques Le Goff (2003), podemos afirmar que la circulación en la Edad Media fue principalmente fluvial y marítima, sin existir buenos transportes terrestres-, lo inédito de Inglaterra, a juicio de Schmitt, es que «transfirió en realidad su existencia de la tierra al elemento marítimo. Con ello no solamente ganó una porción de combates y guerras navales, sino también algo diferente e infinitamente superior: ganó una revolución y de las más grandes, una revolución espacial, de signo planetario». (Schmitt 1952, p. 55-56).

El cambio existencial de Inglaterra, quedó de manifiesto en su nueva interpretación y práctica de la guerra. La lucha marítima dejó de considerarse como un combate cuerpo a cuerpo sobre los barcos —es decir, una extensión de la lucha terrenal-, teniendo ahora como fin el hundimiento del barco enemigo. Por otra parte, el nuevo tipo de guerra incluyó la figura del «bloqueo marítimo», con la cual se dejó a un lado el antiguo modelo del combate específico entre tropas estatales, buscando, a partir de ese momento, efectos comerciales y económicos directos sobre toda la población de los Estados atacados. Ambos cambios de lógicas guerreras - el paso a un combate entre barcos y al objetivo de dominar a toda la población enemiga-, demostraron, genuinamente, que el mar representaba una dimensión novedosa para la lucha espacial (Schmitt 2002, pp. 344-356).

En síntesis, Schmitt plantea que entre los siglos XVI y XVII cambió rotundamente la lucha por la división de los espacios, incorporándose en el ordenamiento espacial mundial un elemento hasta entonces inédito: la existencia 
marítima de los pueblos. Coinciden en este momento histórico, la transformación del nomos y de la imagen humana del espacio, situación aparejada con transformaciones jurídicas, políticas y militares de gran envergadura, que habrían sido dominantes hasta comienzos del siglo XX. Éste es el escenario general que habría determinado la nueva época histórica, generando un cambio «de signo total» - técnico, científico, político, jurídico, militar y cultural— que tuvo alcance planetario.

\section{El contrincante del Behemoth y el Leviathan}

La diferencia entre el dominio de la tierra y del mar es tal, que Schmitt (1952, p. 16) llegó a interpretar la historia universal como «la historia de las luchas entre las potencias marítimas contra las terrestres y de las terrestres contra las marítimas». Este conflicto lo representa a partir de un antiguo mito judío: la pugna entre el Leviathan —una poderosa ballena - y el monstruo terrestre Behemoth — representado como un toro o un elefante-. Según cuenta la interpretación de los Cabalistas, el Behemoth intentó destrozar al Leviathan con cuernos y colmillos, mientras que este último - tal cual sucedería en un bloqueo marítimo - cerró con sus aletas el hocico del monstruo terrestre para evitar que respire y coma (Schmitt 1952, p.17). Pues bien, ¿podría decirse que, de acuerdo con la interpretación de Schmitt, en el último tiempo se ha incorporado un nuevo monstruo a esta pugna de fieras míticas, es decir, un nuevo elemento de lucha por los espacios, que se añadiría al terrestre y al marítimo?

Resolver esta interrogante no es fácil, principalmente porque Schmitt no hizo demasiadas aclaraciones al respecto en su obra. Lo que él inmediatamente propuso fue que, a contar del siglo XX, se podría distinguir un nuevo estadio de la gran revolución espacial, o - lo que le parece bastante probable - una segunda revolución de esa especie (Schmitt 1952, p. 108-109). La guerra mundial de 1914 se habría iniciado con este nuevo signo planetario, el de una renovada vinculación entre el hombre y su espacio vital.

Nuevamente, esta revolución podría interpretarse como la coexistencia de dos fenómenos interrelacionados: nomos y conciencia colectiva. En el nivel nomistico, el rol que tuvieron los descubrimientos de tierra en el siglo XVI podría equipararse al que ahora sostenía la revolución industrial. Lo que hizo la técnica moderna — simbólicamente con el paso desde el velero al barco de vapor, y luego al buque de guerra-, fue insertarse entre el mar y el hombre, obstruyendo el contacto directo que tenían ambos elementos.

«El mar — señala Schmitt (1952, pp.104-105) — siguió conservando su poder forjador de hombres. Pero las últimas consecuencias de aquel poderoso impulso que había transformado un pueblo de pastores en patria de marinos — refiriéndose al caso de Inglaterra - disminuyeron y acabaron por extinguirse. Entre el mar como elemento y la existencia humana vino a interponerse un aparato mecánico. [...] La revolución industrial convirtió a los espumadores de océanos y a los hijos del

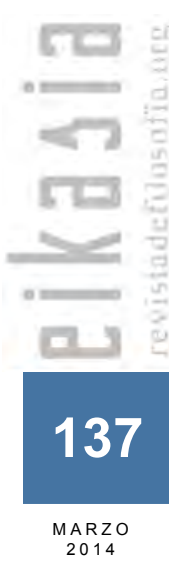


mar en simples constructores de máquinas y servidores de su poder».

El punto neurálgico del vínculo entre hombre y mar había sido afectado por la revolución industrial, proceso que aminoró la inserción vital del hombre en el espacio marítimo. La técnica promovida desde el siglo XX involucraría una especie de mecanización de las acciones humanas, situación aclarada por Schmitt en un diálogo radiofónico que realizó en 1954. En él, el jurista interpretó el uso humano de los recursos tecnológicos, dictaminando: «ya no es el hombre como tal, sino una reacción en cadena desatada por él la que logra todo esto» (Schmitt 2010, p. 48).

En el ámbito de la conciencia colectiva, los avances técnicos en transporte y comunicaciones habrían determinado una transformación del imaginario espacial. En este aspecto, es vital la aparición del aeroplano — progresivamente elaborado desde 1890 con los experimentos de Clément Adert, aunque célebremente mejorado desde 1903 debido a los vuelos sostenidos y controlados de los hermanos Wright-. Aquí, sugiere tentativamente Schmitt, comienza la conquista de un tercer elemento espacial, el aire. Las consecuencias del aeroplano habrían sido múltiples, afectando las comunicaciones, la guerra, las medidas espaciales e incluso el dominio humano:

\begin{abstract}
«Álzase el hombre sobre la superficie del suelo y de las aguas y recibe en sus manos un medio de comunicación de especie totalmente nueva y también un arma nueva por completo. Proporciones y medidas altéranse más aún y las posibilidades de dominio del hombre sobre la Naturaleza se extienden a insospechados dominios» (Schmitt 1952, p. 110)
\end{abstract}

En esta transformación de la imagen espacial, no es menor el impacto que tuvo la electricidad y especialmente la radiofonía, inventada en 1894 por Nikola Tesla y mejorada posteriormente por Guillermo Marconi. Schmitt nota que las ondas radiofónicas cruzan con facilidad los países, pudiendo incluso dar la vuelta al mundo en un instante; ello iría en favor de la hipótesis de que en esta época irrumpió el aire como tercer elemento vital. Si el Behemoth representaba la tierra, y el Leviathan el mar, podría estar sumándose a la batalla un gran pájaro mítico (Schmitt 1952, p. 111).

Lo problemático de este diagnóstico, es que el mismo Schmitt (1952, pp. 110-111) lo considera apresurado. El jurista plantea que algunos de los medios aeronáuticos — como los motores de explosión — podrían indicar un escondido predominio del fuego como elemento determinante de la nueva existencia humana. Por ello, termina acotando sus pretensiones a la formulación de dos ideas más seguras.

En primer lugar, que el cambio actual en el imaginario colectivo sería tan profundo como el producido en los siglos XVI y XVII. Desde el siglo XX se habría empezado a concebir el espacio como un «campo de fuerzas donde despliega el hombre su energía, su actividad y su esfuerzo» (Schmitt 1952, p. 112), siendo por primera vez posible 
pensar — como hizo Heidegger (2003) - que el espacio está en el mundo y no el mundo en el espacio. ${ }^{4}$ En segundo lugar, Schmitt sugiere que este cambio ha transformado genuinamente la relación entre hombre y mar, ya que, debido al auge de los medios de comunicación, empezó a ser posible para cualquier marinero saber en qué coordenadas del mundo se encontraba su barco, situación que - si se compara con la de los antiguos navegantes a vela - representa un cambio sustantivo en la comprensión humana del espacio marítimo. Ambas conclusiones convergen en una gran sentencia: «el viejo nomos se hunde sin duda y con él todo un sistema de medidas, normas y proporciones tradicionales» (Schmitt 1952, p. 113).

No obstante, como se ha recalcado, para Schmitt la existencia de un nomos no es algo contingente, sino necesario, dado que toda coexistencia de pueblos se basa en una división específica del espacio. Por lo tanto, la pregunta fundamental queda abierta: ¿cuál sería en la actualidad el nuevo nomos, es decir, aquel elemento que incita a una lucha por las proporciones planetarias?

Sobre esta problemática, es clave considerar un texto más tardío de Schmitt, presentado en 1978, en el cual el jurista pudo expresar su visión sobre el orden de posguerra: La revolución legal mundial. Allí Schmitt comparó la situación del siglo XVI con la del XX, argumentando que en ambos casos el desarrollo de la estructura económicoindustrial determinó que surgieran interrogantes fundamentales para la humanidad. En el siglo XVI, el avance industrial incitaba a la selección de alternativas teológicas — catolicismo, luteranismo o calvinismo-, y, según la tradicional fórmula de la Paz de Westfalia (1648), la respuesta fue cuius regio, eius religio — quien gobierna impone su religión-. Desde el siglo XX, en cambio, se buscó tomar una decisión sobre qué alternativa políticaliberal-capitalista, socialista-comunista o liberal-socialista - se ajustaba mejor al grado de desarrollo científico, técnico e industrial de los países; en esta época Schmitt considera apropiado el adagio: cuius regio, eius industria —quien gobierna posee la industria—. (Schmitt 2012, pp. 69-70).

La idea que el jurista quiso defender en este escrito, es que el grado de progreso industrial se asocia a un tipo de apropiación planetaria, y que en el período actual de la humanidad éste era la toma de industria:

«La cultura agraria previa había derivado sus categorías de pensamiento del suelo; las conquistas que llevó a cabo eran tomas de tierra porque su auténtica meta era la tierra. En los siglos XVII y XVIII, Inglaterra, el país donde se origina la industrialización moderna, efectuó el tránsito a una existencia marítima y ha logrado dominar el mar «libre» («libre» porque se halla libre de las delimitaciones del suelo); con ello se gestó la toma de mar. A esta etapa sigue ahora la de las tomas de industria. Sólo la posesión de un gran espacio industrial, y ninguna otra cosa, permite hoy en día la toma del espacio mundial» (Schmitt 2012, p. 70).

\footnotetext{
${ }^{4}$ En palabras de Heidegger $(2003$, § 22): «el "mundo circundante" no se inserta en un espacio previamente dado, sino que su mundaneidad específica articula en su significatividad el contexto respeccional de una determinada totalidad de lugares propios circunspectivamente ordenados. Cada mundo particular descubre siempre la espacialidad del espacio que le pertenece».
} 
Como puede apreciarse, en sus escritos tardíos Schmitt se alejó del esquema clásico de los elementos — tierra, agua, aire y fuego - para proponer que lo característico del emergente estadio histórico era el combate por los espacios industriales. El nuevo nomos de la tierra —al menos en un sentido planetario — sería para él la industria, la misma que estaría asociada a la capacidad de gobernar.

Cabe aclarar, que la existencia de un nomos industrial no implica necesariamente que el tipo de guerra dominante utilice estrategias industriales. De hecho, Schmitt sostuvo en numerosas ocasiones la importancia de la aviación en la guerra contemporánea, dando gran relevancia a la naciente experiencia del bombardeo aéreo y al nuevo horizonte guerrero de los pilotos — el cual permite observar, a la vez, las dimensiones terrestre y marítima — (Schmitt 2002, p. 353). Esto último sugiere, que Schmitt no borra del todo al «gran pájaro» dentro del mapa de las transformaciones contemporáneas; sin embargo, sus últimos escritos dejaron entrever que el aire no sería el elemento en disputa que caracteriza al nuevo estadio histórico.

Ante los monstruos míticos Behemoth y Leviathan, finalmente Schmitt habría opuesto una gran cámara de montaje, escoltada, no obstante, por un pájaro bestial. A esta época, caracterizada por las tomas de industria y el desarrollo técnico mecanizante, subyacería una imagen física del espacio, que presenta como un campo de fuerzas en donde el hombre despliega su energía, actividad y esfuerzo. 
Pablo Beytía | La lucha contemporánea por el espacio en la obra de Carl Schmitt

\section{Fuentes bibliográficas}

Arendt, H. (2005). La condición humana. Barcelona: Paidós.

Aristóteles (2003). Metafisica. Buenos Aires: Andrómeda.

Durkheim, É. (2008). Las reglas del método sociológico. Buenos Aires: Losada.

Ferguson, N. (2012). Civilización. Occidente y el resto. Barcelona: Debate.

Heidegger, M. (2003). Ser y Tiempo. Madrid: Trotta.

Le Goff, J. (2003). En busca de la Edad Media. Barcelona: Paidós.

Schmitt, C. (1952). Tierra y Mar. Madrid: Instituto de Estudios Políticos.

Schmitt, C. (2002). El nomos de la tierra. Granada: Comares.

Schmitt, C. (2006). El concepto de lo político. Madrid: Alianza.

Schmitt, C. (2010). Diálogo sobre el poder y el acceso al poderoso. Buenos Aires: Fondo de Cultura Económica de Argentina.

Schmitt, C. (2012). La revolución legal mundial. Buenos Aires: Hydra.

Voigt, R. (2012). De la legitimidad a la legalidad: la revolución legal mundial. En: Schmitt, C. (2012). La revolución legal mundial. Buenos Aires: Hydra.

Weber, Max. 2004. Ética protestante. Buenos Aires: Gradifco. 
